\title{
Numerical simulations of bar percussion instruments
}

\author{
V. DOUTAUT and A. CHAIGNE
}

Groupe Acoustique, Département Signal, URA 820 du CNRS, Télécom Paris, 46 rue Barrault, 75634 Paris cedex 13, France

\begin{abstract}
A numerical model of vibrating bars is developed in order to investigate the behavior of mallet percussion instruments in the time domain. This paper focuses on two major characteristics of such instruments : the nonlinear interaction between the bar and mallet, and the fine tuning of the bar. The latter feature is obtained by simulating a bar with variable section, by means of an implicit finite difference scheme. Simulated bar velocity waveforms and spectra are compared with measurements on real intruments. Auditory evaluation of the model is carried out for different bar materials, geometries, and conditions of impact.
\end{abstract}

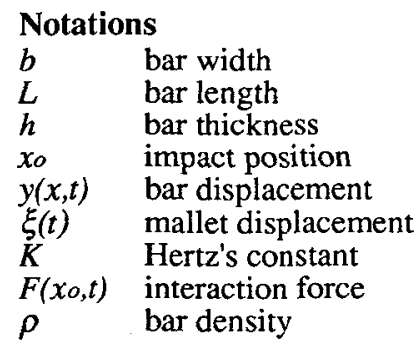

$\begin{array}{ll}E & \text { Young's modulus of the bar } \\ N & \text { number of spatial steps } \\ \Delta x & \text { spatial step } \\ V_{m o} & \text { initial mallet velocity } \\ f_{e} & \text { sampling frequency } \\ \eta & \text { viscoelastic constant } \\ \mu & \text { reduced mass of bar and mallet } \\ \delta & =\left|y\left(x_{o}, t\right)-\xi(t)\right| \text { (compression) } \\ M m & \text { "equivalent" mallet mass }\end{array}$

\section{INTRODUCTION}

Numerical simulations can be viewed as intermediate steps between analytical modeling and experimental testing. Such techniques make it possible to describe the mechanical behavior of complex structures, even if nonlinear phenomena are involved, as is the case for the excitation of almost all classes of percussive instruments. Only in very strict cases can the validity of a given numerical model be tested by comparing the results of a simulation with analytical solutions. The results of a numerical modeling provide us with a rough approximation of real structures, but its main interest lies more in its particular ability to highlight the major outlines of physical phenomena. However, a numerical model needs to be validated through comparisons with experimental data. The main difficulty is that experimental signals are often corrupted by complicating factors which are not taken into account by the model, a consequence of the complexity of real structures.

In a previous work by the authors, the vibrations of xylophone bars were approximated by a 1-D modeling of transverse bending waves, which contribute primarily to the sound pressure 
field [1]. In these numerical experiments, an explicit scheme was used to model a wooden bar with constant section, including the influence of structural damping. Although this simple model can reproduce convincingly xylophone vibrations, and allows the unambiguous recognition of this instrument, it still suffers from major limitations. First, it has been shown that the spatial step $\Delta x$, in the case of explicit finite difference schemes, cannot be less than about $10 \mathrm{~mm}$, for stability reasons. As a consequence, the position and spatial extent of the impact cannot be defined with great accuracy. Second, it is necessary to simulate a bar with a variable section, in order to obtain a well-defined pitch [2]. Here again, the section profile needs to be given with sufficient precision, so as to carefully adjust the frequencies of the first partials.

These questions concerning the excitation and fine tuning of the bar form the basis of the present paper. A nonlinear model for the interaction between bar and mallet, based on the well-known Hertz's law of impact [3], is presented in Section 2. Within the framework of this theory, our contribution mainly consists of elaborating experimental procedures, in order to extract the Hertz's constant $K$ from impact measurements on real instruments [4]. In Section 3, an implicit finite difference scheme is presented, making it possible to approximate variable sections with great accuracy, because of the good stability properties of the numerical algorithm. The results are illustrated by comparing simulated and measured bar velocity waveforms, both in the time and frequency domain.

\section{BAR-MALLET INTERACTION}

The bar-mallet interaction generates sound and vibrations which are responsible for the initial transients of percussive instruments. That percussionists usually take great care in selecting their mallets indicates strongly that these transients are perceptually significant. In order to discriminate between various mallets commonly used by musicians, Bork performed measurements of shock spectra on force transducers [5]. The present investigation is conducted in the time-domain, with mallets striking real bars. It is assumed that the impact force is conveniently described by Hertz's law :

$$
F=K \delta^{1.5}
$$

The experimental method then consists of extracting both the equivalent mass $M_{m}$ of the mallet, and Hertz's constant $K$, from measurements of the contact force, velocity and acceleration of the mallet. This method will be now briefly summarized.

An accelerometer is fixed near the head of the mallet, and strikes first a rigidly fixed impedance head. The equivalent mass is obtained by computing the ratio between the force signal delivered by the impedance head and the acceleration, in both the time and frequency domain. This "equivalent" mass is not equal to the complete mass of the mallet, since the exciter is held at one end by the hand of the player. In practice, it was found that $M_{m}$ is roughly $20 \%$ greater than the mass of the mallet's head.

In a second series of experiments, the mallet strikes a bar with free ends, at a given impact position, approximating the normal use of the instrument. In practice, the assumption of free ends for the boundary conditions is achieved by a weak coupling with lossy springs. During the impact, the energy balance can be written [3] :

$$
\frac{1}{2} \mu V_{m o}^{2}=\frac{1}{2} \mu\left(\frac{d \delta}{d t}\right)^{2}+\frac{2}{5} K \delta^{5 / 2}
$$

Solving Eq. (2) yields the duration of contact :

$$
\tau=3.21\left(\frac{\mu^{2}}{K^{2} V_{m o}}\right)^{1 / 5}
$$


This duration $\tau$ is obtained from measurements of the mallet's acceleration. Integrating this signal yields the mallet's velocity, from which $V_{m o}$ can be derived. The Hertz's constant is then calculated by inverting Eq. (3). This constant depends on the geometry, and the elasticity parameters, of both the mallet and bar. For a rosewood bar struck with a mallet's head made of boxwood, with initial velocity equal to $2.1 \mathrm{~m} / \mathrm{s}$, for example, the measured contact duration is $0.16 \mathrm{~ms}$, which yields $K=4.110^{8} \mathrm{~N} / \mathrm{m}^{1.5}$.

\section{A NUMERICAL MODEL FOR A BAR WITH VARIABLE SECTION}

As described in earlier studies devoted to mallet instruments, one technique used by makers, in order to tune the first modes of vibration, consists of cutting an arch in the underside of the bar [2]. Orduna-Bustamente has shown recently that the profile of this arch can be reasonably approximated by a parabolic shape. The two parameters of this parabola are related to the desired frequency shift of modes 2 and 3 relative to the frequency of the lowest mode, these first three modes together playing a major role in the definition of pitch [6]. Thus, the equation governing the flexural vibrations of such bars with variable thickness $h(x)$, and structural damping $\eta$, becomes :

$$
\frac{\partial^{2} y(x, t)}{\partial t^{2}}=-\frac{E}{12 \rho} \frac{1}{h(x)} \frac{\partial^{2}}{\partial x^{2}}\left[h^{3}(x) \frac{\partial^{2} y(x, t)}{\partial x^{2}}+h^{3}(x) \frac{\eta}{E} \frac{\partial^{3} y(x, t)}{\partial t \partial x^{2}}\right]+f\left(x, x_{o}, t\right)
$$

where $f(x, x o, t)$ is a force density term proportional to the impact force. Eq. (4) has no analytical solution, since it involves nonlinear terms, and, therefore, a numerical approach is required. In order to reproduce the transients convincingly, it was decided to solve directly Eq. (4) in the time domain, using finite differences (FD). In a previous paper, we showed that explicit FD schemes, though leading to simple and efficient algorithms in terms of computation time, require large values for the spatial step $\Delta x$, typically $10 \mathrm{~mm}$, for stability reasons. These values are not compatible with the spatial resolution needed to define both excitation and thickness shape with sufficient precision, within the context of mallet instruments. However, by using appropriate implicit FD schemes, and, in particular, a socalled unconditionally stable scheme, it becomes possible to circumvent this drawback. In this case, it can be shown that the time step $\Delta t$ (i.e., the sampling frequency $f_{e}=1 / \Delta t$ ) and the spatial step can be selected independently. It is interesting to notice here that the selected sampling frequency is related to the estimation of the eigenfrequencies when the bar vibrates freely, whereas a small spatial step is a necessary condition for modeling the bar-mallet interaction during the initial transient. In our numerical experiments, a sampling frequency and spatial step equal to $192 \mathrm{kHz}$, and $1 \mathrm{~mm}$, respectively, were used.

The major idea of the selected implicit scheme consists in approximating the spatial derivatives in Eq. (4) by a mean value of FD terms at time $n+l$ and $n-1$, instead of time $n$, as it is the case for explicit schemes [7]. Thus, the discrete version of Eq. (4) becomes :

$$
\begin{array}{r}
y_{i}^{n+1}+\frac{\sigma^{2}}{2 h_{i}}\left(1+\frac{\eta f_{e}}{E}\right)\left[\frac{1}{h_{i+1}^{3}} y_{i+2}^{n+1}-2\left(\frac{1}{h_{i+1}^{3}}+\frac{1}{h_{i}^{3}}\right) y_{i+1}^{n+1}+\left(\frac{1}{h_{i+1}^{3}}+4 \frac{1}{h_{i}^{3}}+\frac{1}{h_{i-1}^{3}}\right) y_{i}^{n+1}-2\left(\frac{1}{h_{i}^{3}}+\frac{1}{h_{i-1}^{3}}\right) y_{i-1}^{n+1}+\frac{1}{h_{i-1}^{3}} y_{i-2}^{n+1}\right] \\
=2 y_{i}^{n}-y_{i}^{n-1}-\frac{\sigma^{2}}{2 h_{j}}\left(1-\frac{\eta f_{e}}{E}\right)\left[\frac{1}{h_{i+1}^{3}} y_{i+2}^{n-1}-2\left(\frac{1}{h_{i+1}^{3}}+\frac{1}{h_{i}^{3}}\right) y_{i+1}^{n-1}+\left(\frac{1}{h_{i+1}^{3}}+4 \frac{1}{h_{i}^{3}}+\frac{1}{h_{i-1}^{3}}\right) y_{i}^{n-1}-2\left(\frac{1}{h_{i}^{3}}+\frac{1}{h_{i-1}^{3}}\right) y_{i-1}^{n-1}+\frac{1}{h_{i-1}^{3}} y_{i-2}^{n-1}\right]
\end{array}
$$

where $\sigma^{2}=E / 12 \rho$, and where $i$ and $n$ are the space and time index, respectively.

Table I : Values of the parameters used for the simulations

\begin{tabular}{|llll|}
\hline BAR & $b=3.9 \mathrm{~cm}$ & $L=27.4 \mathrm{~cm}$ & $x_{o}=\mathrm{L} / 3$ \\
(Rosewood) & $E=2.1310^{10} \mathrm{~N} / \mathrm{m}^{2}$ & $\eta=5.010^{3} \mathrm{~N} . \mathrm{s} / \mathrm{m}^{2}$ & $\rho=1015 \mathrm{Kg} / \mathrm{m}^{3}$ \\
MALLET (Boxwood) & $M m=11.2 \mathrm{~g}$ & $K=4.110^{8}$ \\
SAMPLING & $\mathrm{f}_{\mathrm{c}}=192 \mathrm{kHz}$ & & $\mathrm{N}=274(\Delta \mathrm{x}=1.0 \mathrm{~mm})$ \\
\hline
\end{tabular}


(a)

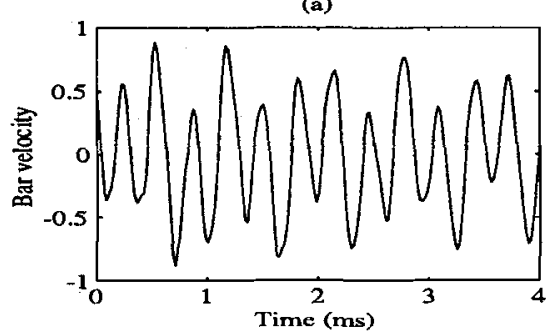

(c)

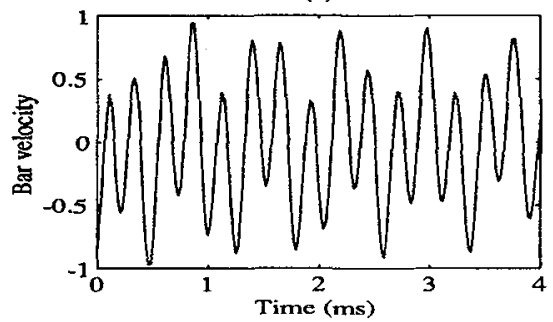

(b)

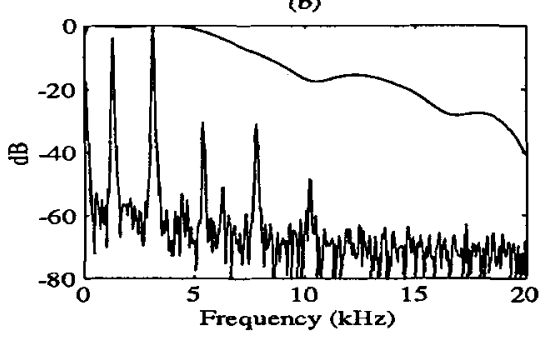

(d)

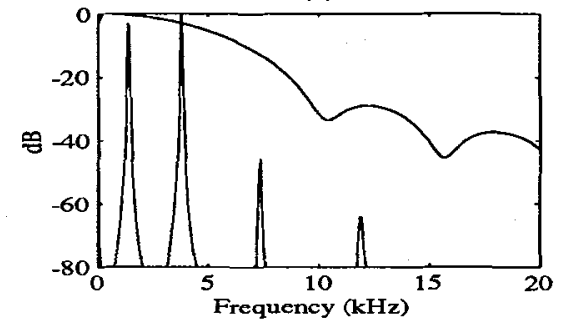

Fig. 1 : Comparisons between measured $(a, b)$ and simulated $(c, d)$ xylophone bar velocity waveforms and spectra. The vertical units are normalized with respect to the maximum (i.e., to 1 for the waveforms and $0 \mathrm{~dB}$ for the spectra). The spectral envelopes for measured and simulated impact forces are displayed in Fig. 1-b, and Fig. 1-d, respectively.

Fig. 1 shows a comparison between measured and simulated xylophone bar velocity waveforms and spectra, during the first $4 \mathrm{~ms}$ of the signals, for a rosewood bar with constant section. It can be seen that the spectral envelopes for the interaction force are very similar, and that the magitude of the two first partials are well reproduced. However, the velocity spectra differ significantly for frequencies above $5 \mathrm{kHz}$, a consequence of the 1-D approximation of thin bars in the physical model used for the simulations. The two waveforms present similar patterns, which is confirmed by auditory evaluation, even if a closer look shows obvious differences. Similar results are obtained for a bar with variable section.

\section{Acknowlegments}

This work was partially supported by a grant from the French Ministry of Culture (Direction de la Musique et de la Danse). The authors wish to thank the French Company Rythmes \& Sons for its help in supplying xylophone bars, and Dr. Stephen Lakatos for his assistance in revising an earlier version of this text.

\section{References}

[1] Doutaut V. \& Chaigne A. (1993), "Time domain simulations of xylophone bars", SMAC 1993, Publications of the Royal Swedish Academy of Music (to appear).

[2] Bork I. (1983), "Zur Abstimmung und Kopplung von schwingenden Stäben und Hohlraumresonatoren", Dissertation, Techn. Univ. Carolo-Wilhelmina, Braunschweig.

[3] Landau L. \& Lifchitz L. (1967), Théorie de l'élasticité, Mir, Moscow (in French).

[4] Bérard Ph. (1993), "Modèle d'excitateur pour la synthèse sonore", Mémoire de fin d'études, TELECOM Paris (unpublished).

[5] Bork I. (1990), "Measuring the acoustical properties of mallets", Applied Acoustics 30, 207-218.

[6] Orduna-Bustamente F. (1991), "Nonuniform beams with harmonically related overtones for use in percussion instruments", J. Acoust. Soc. Am. 90(6), 2935-2941.

[7] Crandall, S.H. (1954), "Numerical treatment of a fourth-order parabolic partial differential equation", J. Assoc. for Computing Machinery, Vol.1, 111-118. 